\title{
Retrieval of optically thin cloud emissivity from brightness temperatures provided by IR Camera of JEM-EUSO Mission
}

\author{
Susana Briza , Antonio J. de Castro, Isabel Fernández-Gómez, Irene Rodríguez, and Fernando López for JEM-EUSO \\ Collaboration \\ Universidad Carlos III de Madrid, Physics Department, Avda. de la Universidad 30, 28911 Leganés (Madrid), Spain
}

\begin{abstract}
Clouds interact with the radiation propagating through the atmosphere absorbing, reflecting and transmitting part of the energy. This interaction may lead to misinterpretation of data retrieved from the radiation observed by JEM-EUSO UV telescope. JEM-EUSO Mission includes an Atmospheric Monitoring System (AMS), consisting of a LIDAR and an IR Camera, devoted to provide the cloud coverage and the cloud height in the FOV of the main UV Telescope. Different methods can be applied to retrieve the cloud top height from IR images. Radiative algorithms are based on the Radiative Transfer Equation which changes significantly depending on the cloud optical depth (thick or thin clouds). The cloud temperature retrieval becomes much more difficult for thin ones. In this work we present a methodology based on brightness temperatures in 10.8 and $12 \mu \mathrm{m}$ bands measured by the JEM-EUSO IR camera. The method uses Look Up Tables (LUTs). It has been validated with data obtained by simulation but also in real scenarios (MODIS images). The results are very promising for emissivities higher than 0.5. For lower emissivities, the retrievals become much more difficult since the IR radiation impinging the IR Camera also comes from other emitters (Earth surface and atmosphere beneath the cloud).
\end{abstract}

\section{Introduction}

The aim of JEM-EUSO Mission is to identify the sources of Ultra High Energy Cosmic Rays (UHECR) [1] from the International Space Station. However JEM-EUSO telescope will not observe UHECRs directly but instead it will detect the UV radiation produced by the interaction between the UHECRs and the atmosphere. The analysis of the UV images, the Extensive Air Shower (EAS) reconstruction, will give information of the UHECR properties (energy, arrival direction, etc.).

However the UV radiation is affected by the atmosphere and the clouds in the path between the EAS and the telescope which can lead to misreconstruction of the EAS event [2]. Therefore it is extremely important to know the atmospheric conditions and detect if there are clouds in the Field of View (FoV) of the telescope. Moreover, the cloud top height and the cloud emissivity are essential for an appropriate reconstruction of the EAS.

In order to monitor the required information in the main telescope FoV the JEM- EUSO mission will implement an Atmospheric Monitoring System (AMS) which will be attached to the main system. The AMS consists of a LIDAR, an infrared (IR) camera and global atmospheric models [3]. The LIDAR will measure the optical depth profiles of the atmosphere in selected directions [4]. The IR camera will provide the cloud coverage and the cloud temperature. The global atmospheric models will be used to retrieve the atmospheric parameters (temperature, pressure and humidity vertical profiles) in the monitored region.

Cloud Top Height (CTH) retrieval can be performed using radiometric information or stereovision technique, although this paper focuses on the first technique. The

a e-mail: sbriz@fis.uc3m.es radiation emitted by the cloud is basically related to its emissivity and temperature according to Planck's law. The radiance emitted by the cloud is measured by the sensor (IR camera) and the system retrieves the temperature of the cloud from the radiance measurement. Therefore the radiation received by the IR camera can be used to get the cloud top temperature (CTT) and then estimate the cloud height by using the relationship between the temperature and the height. Atmospheric soundings available in the area and global atmospheric models will help to obtain the $\mathrm{CTH}$ from the cloud temperature and the temperature vertical profile.

However, due to atmospheric effects (gas absorption/emission); the IR radiance emitted by the cloud is not the one received by one by the IR camera. Therefore the temperature retrieved from measured radiance (Brightness Temperature, BT) is not the temperature of the cloud. Moreover, if the cloud emissivity is lower than 1, the radiance emitted by the cloud is not exactly the corresponding radiance emitted by a blackbody at the same temperature and then it is necessary to know the emissivity in advance to retrieve the CTT from the BT. Hence it is clear that some algorithms are necessary to correct the atmospheric effects, to calculate the emissivity and then to obtain the cloud temperature from BT.

In a previous work, a simplified algorithm was designed and validated. This Split Window Algorithm (SWA) was based on the bi-spectral design of the JEM-EUSO IR camera which measures the BT in two $1-\mu \mathrm{m}$ width bands centred at 10.8 and $12 \mu \mathrm{m}([5,6])$. The algorithm was calculated with an approximation of the Radiative Transfer Equation, considering the maximum emissivity $(\varepsilon=1)$ and simulations of atmospheric radiance with different vertical atmospheric profiles of temperature, humidity, etc. The SWA was validated with simulated scenarios in different atmospheric conditions. The error

This is an Open Access article distributed under the terms of the Creative Commons Attribution License 4.0, which permits unrestricted use, distribution, and reproduction in any medium, provided the original work is properly cited. 
was higher for low clouds and humid atmospheres but even so it was lower than $0.3 \mathrm{~K}$. The SWA was also applied to real scenarios (MODIS images in spectral bands 31 and 32). The difference between the temperature retrieved by the SWA and this one provided by MODIS was lower than $1 \mathrm{~K}$ for thick water clouds $(\varepsilon=1)$ [6]. However for thin clouds $(\varepsilon<1)$ the difference is higher, as expected since the SWA was calculated under the hypothesis $(\varepsilon=1)$. The work presented here is focused on the methodology developed to retrieve the cloud emissivity from the BT given by the IR Camera in two spectral bands. Our main objective is to design and evaluate a specific methodology for the JEM-EUSO IR camera to calculate the emissivity of the clouds. This cloud parameter will allow us to retrieve the CTT by the SWA if the emissivity is 1 (thick clouds) or other algorithm if the emissivity is lower than 1 (thin clouds). The methodology will be described in Sect. 2 and the results of its validation in simulated and real scenarios will be explained in Sect. 3 .

\section{LUT methodology}

The design of JEM-EUSO IR Camera is constrained by JEM-EUSO requirements which have led to a bi-spectral camera with two $1 \mu \mathrm{m}$-width bands centered at 10.8 and $12 \mu \mathrm{m}$ [5]. After calibration, the IR Camera will provide the BT in both bands. The small emissivity difference in both bands is translated into small differences in BT which will be used to retrieve the emissivity. The methodology is based on the idea of Look Up Table (LUT) which combines, in this case, the difference of BT in both bands with the BT in $10.8 \mu \mathrm{m}$ band and the emissivity. The BTs needed to build the LUT have been calculated from simulations of different IR scenarios (different clouds and atmospheric conditions) by using the Radiative Transfer Equation (RTE) and an atmospheric simulation code (MODTRAN). In Sect. 2.1 the process to generate the LUT is described. The procedure to apply the LUT to retrieve the emissivity from BTs in both bands is explained in Sect. 2.2.

\subsection{LUT generation procedure}

In order to generate the BT values in different scenarios, radiance simulations with clouds at different heights and with different emissivities have been performed. The simulation process involves:

a) Defining IR scenarios: the emitters (Earth surface, cloud and atmosphere) and interfering atmosphere.

b) Applying a radiative model to different IR scenarios to obtain the spectral radiance at sensor height.

c) Retrieving the BTs in both bands from the spectral radiance previously calculated.

As explained above, to simulate the spectral radiance at the sensor height we apply a radiative model to an IR scenario (model inputs). To run the model it is necessary to take into account the radiative effects of the following elements:

a) The Earth surface, which is characterized by a surface temperature Ts and a surface emissivity $\varepsilon$ s.

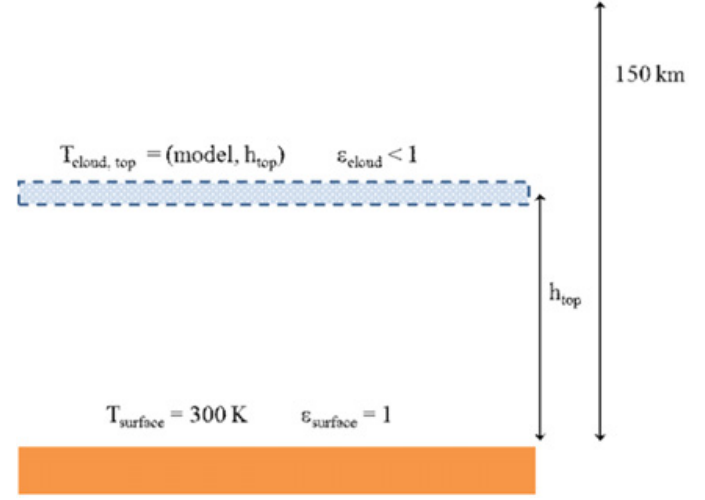

Figure 1. Components of the radiative model.

b) The cloud, modeled as a plane parallel and homogeneous surface that represents the cloud radiative properties (temperature and emissivity).

c) The atmospheric model, in which the atmosphere is split in layers at different heights where a value of temperature, pressure and gas concentration has to be assigned. For this study, the atmosphere has been divided in $0.5 \mathrm{~km}$ thick layers from the bottom to the top of the atmosphere, whose thickness is considered as $100 \mathrm{~km}$. Far away from this height the atmosphere has no effects on the infrared calculations. In this way, the atmospheric model is described by vertical profiles of temperature, pressure and concentrations. The basic atmospheric models used in this work are widely used in the atmospheric physics field and represent seasonal average values for different latitudes.

Model 1: Tropical model $\left(15^{\circ} \mathrm{N}\right)$

Model 2: Midlatitude Summer (45 ${ }^{\circ}$, July)

Model 3: Midlatitude winter ( $45^{\circ} \mathrm{N}$, January)

Model 4: Subartic Summer $\left(60^{\circ} \mathrm{N}\right.$, July)

Model 5: Subartic Winter ( $60^{\circ} \mathrm{N}$, January)

Model 6: 1976 US Standard Atmosphere.

The radiative model consists of the Earth surface at certain temperature; the atmosphere and a cloud located at a certain altitude (See Fig. 1). The radiative transfer equation (Eq. (1)) provides the spectral radiance at the entrance pupil of the sensor located at an altitude of $400 \mathrm{~km}$ (ISS height) for the nadir line of sight. The radiance coming from the surface is partially absorbed by the atmosphere and the cloud (first term of the Eq. (1)). The atmosphere between the surface and the cloud emits radiation accordingly to its temperature and the radiation is absorbed in part by the cloud and the atmosphere above (second term of Eq. (1)). Of course, the cloud emits energy that is absorbed in part by the atmosphere (third term of the Eq. (1)). Finally the atmosphere between the cloud and the sensor also emits energy (fourth term of Eq. (1)).

$$
\begin{aligned}
L(v)= & \varepsilon_{S} B\left(T_{s}, v\right) \tau_{0}^{C T H}(v)\left(1-\varepsilon_{c}\right) \tau_{C T H}^{150}(v) \\
& +L_{0}^{C T H}(v)\left(1-\varepsilon_{c}\right) \tau_{C T H}^{150}(v) \\
& +\varepsilon_{c} B\left(T_{C T H}, v\right) \tau_{C T H}^{150}(v)+L_{C T H}^{150}(v) .
\end{aligned}
$$




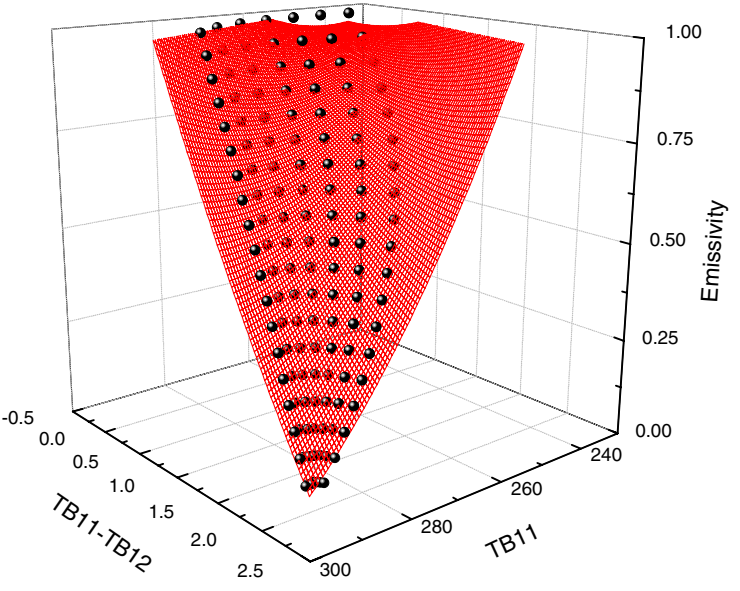

Figure 2. Example of LUT generated with a Tropical atmospheric model.

Where:

$\mathrm{B}(\mathrm{T}, \nu)$ is the blackbody radiance given by Planck's law at temperature $\mathrm{T}$ and wavelength $v$.

Ts and $\mathrm{T}_{\mathrm{CTH}}$ are the surface temperature and the cloud top temperature, respectively.

$\varepsilon_{s}$ is the emissivity of the Earth surface.

$\varepsilon_{c}$ is the emissivity of the cloud.

$\tau_{0}^{C T H}(v)$ is the atmospheric transmittance between the surface and the cloud top height (CTH).

$\tau_{C T H}^{150}(v)$ is the atmospheric transmittance between CTH and the sensor.

$L_{0}^{C T H}(v)$ is the radiance of the atmosphere between the surface and CTH.

$L_{C T H}^{150}(v)$ is the radiance of the atmosphere between $\mathrm{CTH}$ and the sensor.

The atmospheric transmittances and radiances between the cloud and the IR camera were calculated by the wellknown Modtran code (version 5). This procedure was repeated for different clouds changing their temperature (height) and their emissivity to get a sufficiently large set of BTs to allow us to generate the LUT. The LUT consists of a set of points whose components are $\left(\mathrm{BT}_{11}-\mathrm{BT}_{12}\right.$, $\left.\mathrm{BT}_{11}, \varepsilon\right)$.

\subsection{LUT application procedure}

The BTs given by the JEM-EUSO IR camera would be the LUT inputs. Then the emissivity would be retrieved from the LUT by interpolation. In order to make easier the interpolation process when applied to a huge number of points, an image for instance, the $\left(\mathrm{BT}_{11}-\mathrm{BT}_{12}, \mathrm{BT}_{11}\right.$, $\varepsilon)$ values have been fitted to a surface. In this way the emissivity is expressed as a function of the inputs $\left(\mathrm{BT}_{11^{-}}\right.$ $\mathrm{BT}_{12}, \mathrm{BT}_{11}$ ). In Fig. 2 an example of LUT is shown. The LUT points (black points) have been calculated with the procedure previously explained in Sect. 2.1. The atmospheric model used for the simulations has been the Tropical Model.

From Fig. 2 it is deduced that the BTs differences are very low for low emissivities. This fact implies that the resolution of the IR camera should be very high to be able to retrieve low emissivities.
Table 1. Examples of IR scenarios simulated for validation purposes and its emissivity retrieval error. Scenarios correspond to a cloud located at $4.5 \mathrm{~km}$ and $0.5 \mathrm{~km}$ thickness. The cloud emissivity is different for each scenario.

\begin{tabular}{|c|c|l|l|l|c|}
\hline $\mathbf{L W C}\left(\mathbf{g} / \mathbf{m}^{\mathbf{3}}\right)$ & $\varepsilon_{\text {Csimul }}$ & $\mathbf{B T}_{\mathbf{1 1}}(\mathbf{K})$ & $\mathbf{B T}_{\mathbf{1 2}}(\mathbf{K})$ & $\varepsilon_{\text {Cretr }}$ & $\Delta \varepsilon$ \\
\hline 0.1 & 1 & 273.43 & 273.28 & 1.00 & 0 \\
\hline 0.05 & 0.96 & 274.44 & 274.12 & 0.94 & 0.02 \\
\hline 0.03 & 0.83 & 276.62 & 275.92 & 0.80 & 0.03 \\
\hline 0.02 & 0.73 & 279.36 & 278.25 & 0.65 & 0.08 \\
\hline 0.01 & 0.48 & 284.70 & 282.95 & 0.40 & 0.08 \\
\hline 0.005 & 0.28 & 288.99 & 286.85 & 0.23 & 0.05 \\
\hline
\end{tabular}

Other LUTs have been built for other atmospheric models (US Standard 1976 and Midlatitude Summer).

\section{LUT validation}

In order to test the capabilities of this methodology the LUT has been validated with simulations and also tested in real scenarios. Results of validations are explained in Sect. 3. The advantage of this validation is that the emissivities of the cloud are obviously known since they are inputs of the simulations. Therefore the comparison between the emissivity retrieved by the LUT and this one used as input can be considered the error of the LUT-based retrieval methodology. Additionally the LUT methodology has been applied to real scenarios (MODIS images) and compared with the MODIS emissivity product, which is described in Sect. 3. Although the MODIS products are also estimates the comparison between the emissivities retrieved by the LUT and the emissivities given by MODIS is an acceptable procedure to study the LUT methodology performance in real scenarios.

\subsection{LUT validation in simulated scenarios}

For validation purposes, some IR scenarios have been simulated following the procedure explained in Sect. 2 but the clouds parameters $\left(\mathrm{H}, \mathrm{T}_{\mathrm{C}}, \varepsilon_{\mathrm{C}}\right)$ and the atmospheres used for the validation are different to these ones used for the LUT. An example of a cloud at $4.5 \mathrm{~km}$ with different emissivities is shown in Table 1. The cloud physical parameters concerning the emissivity are the cloud thickness and the Liquid Water Content (LWC). In this example the cloud thickness is fixed at $0.5 \mathrm{~km}$ and the LWC is changed from $0.0005 \mathrm{~g} / \mathrm{m}^{3}$ to $0.1 \mathrm{~g} / \mathrm{m}^{3}$ to get cloud emissivities from 0.28 to 1 respectively. In this Table 1 the BTs in both bands $\left(\mathrm{BT}_{11}\right.$ and $\left.\mathrm{BT}_{12}\right)$ obtained from simulations corresponding to these cloud conditions are also presented. After applying the LUT procedure, using these BTs as inputs, the emissivity is retrieved $\left(\varepsilon_{\text {Cretr }}\right)$ and compared with the emissivity used in the simulations. The difference between both emissivities gives us the error in the emissivity retrieval $(\Delta \varepsilon)$, also in Table 1 .

Although errors increase when emissivity decreases, emissivity retrieval errors keep below 0.08 which is a very promising result. Even for low emissivities $(0.28)$ the error is below $18 \%$. 
Table 2. Emissivity retrieval errors for examples of IR scenarios involving clouds at different heights.

\begin{tabular}{|c|c|c|c|c|c|c|}
\hline $\mathbf{H}(\mathbf{k m})$ & $\mathbf{T}_{\text {Csimul }}(\mathbf{K})$ & $\varepsilon_{\text {Csimul }}$ & $\mathbf{B T}_{\mathbf{1 1}}(\mathbf{K})$ & $\mathbf{B T}_{\mathbf{1 2}}(\mathbf{K})$ & $\varepsilon_{\text {Cretr }}$ & $\Delta \varepsilon$ \\
\hline 5 & 270.4 & 1 & 270.1 & 269.9 & 0.96 & 0.04 \\
\hline 5 & 270.4 & 0.9 & 272.7 & 272.2 & 0.87 & 0.03 \\
\hline 5 & 270.4 & 0.8 & 275.8 & 274.4 & 0.68 & 0.12 \\
\hline 5 & 270.4 & 0.7 & 277.8 & 276.5 & 0.61 & 0.09 \\
\hline 7 & 262.7 & 1 & 262.5 & 262.5 & 1.00 & 0 \\
\hline 7 & 262.7 & 0.9 & 266.2 & 265.7 & 0.86 & 0.04 \\
\hline 7 & 262.7 & 0.8 & 269.9 & 268.8 & 0.75 & 0.05 \\
\hline 7 & 262.7 & 0.7 & 273.0 & 271.8 & 0.65 & 0.05 \\
\hline
\end{tabular}

Other examples at different heights $(5$ and $7 \mathrm{~km})$ are shown in Table 2 . The heights $(\mathrm{H})$, temperatures $\left(\mathrm{T}_{\text {Csimul }}\right)$ and emissivities $\left(\varepsilon_{\text {Csimul }}\right)$ used in the simulations are shown in Table 2. $\mathrm{BT}_{11}$ and $\mathrm{BT}_{12}$ obtained from simulations and used as inputs in the LUT are also included in the table. Finally the retrieved emissivity and the retrieval error are also presented in Table 2. The comparison between Tables 1 and 2 reveals that there is not a clear relation between emissivity retrieval errors and the cloud height, in fact, they do not change drastically with the cloud height. Moreover, in general it can be stated that emissivity retrieval errors are lower than 0.1 .

\subsection{LUT application in real scenarios (MODIS images)}

The LUT-based methodology has been applied to MODIS BT images of bands 31 and 32 in order to evaluate the performance of this proposal to retrieve the cloud emissivity. As mentioned above, the advantage of using MODIS sensor is that it provides many products such as cloud temperature, phase, emissivity, etc. These products can be used to compare our retrievals cloud temperature and emissivity to evaluate their performance. The selected image is over the Southern Hemisphere (Pacific Ocean) and its coordinates are Latitude [40S, 60S] and Longitude $[160 \mathrm{~W}, 120 \mathrm{~W}]$. The image was taken on 16/05/2012 at 09:55 UTC.

In Fig. 3 the cloud temperature and phase images given by MODIS are shown (upper image and lower images respectively). In the temperature image, red colour means warm clouds and therefore low clouds. Blue colour means cold clouds and consequently high clouds. White pixels correspond to clear sky. In cloud phase image blue, cyan, yellow and red designate water, ice, mixed and uncertain cloud phase. The combined analysis of these images reveals that the scene is mostly covered by low water clouds. However some ice clouds appear over some areas located at the lower part of the image. Some small areas of ice clouds can be also appreciated in the northern part.

Applying the LUT to the BTs provided by MODIS in the bands 31 and 32 , centred at 10.8 and $12 \mu \mathrm{m}$ respectively, the emissivity is retrieved. To evaluate the result the emissivity retrieved and the MODIS emissivity image are subtracted. In Fig. 4, the upper image shows the emissivity difference image where green, blue and red depict $0,-1$ and 1 emissivity differences respectively.

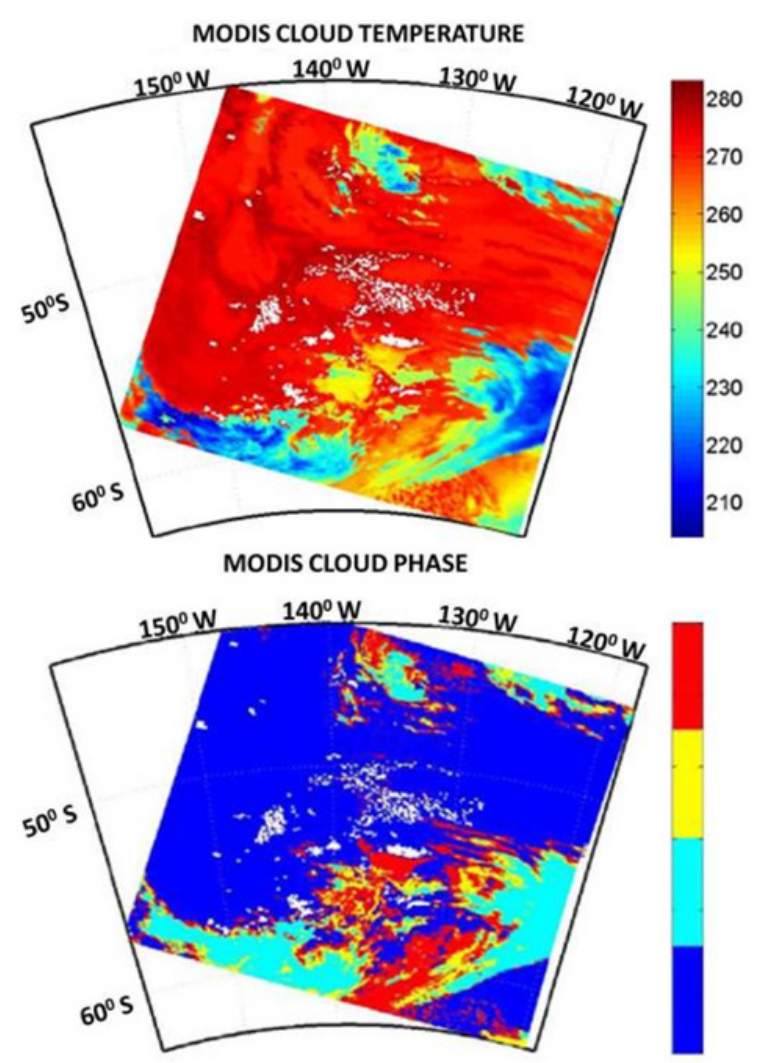

Figure 3. Upper image: cloud temperature (MODIS product). Lower image: cloud phase (MODIS product) where blue, cyan, yellow and red means water, ice, mixed and uncertain cloud phase respectively.

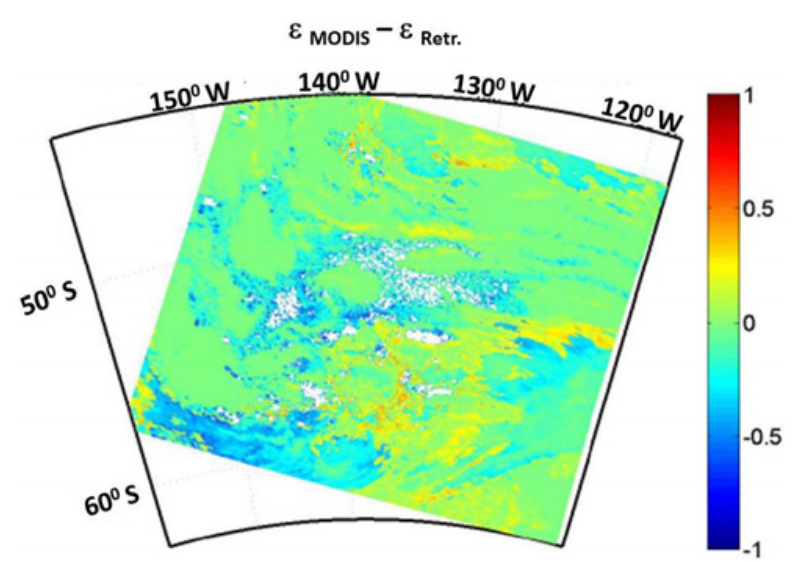

Figure 4. Difference between the emissivity retrieved by the LUT procedure and that provided by MODIS. Green colour indicates 0 difference and dark blue and red maximum difference.

The Fig. 4 clearly shows that the emissivity differences are close to 0 , which means that the emissivity retrieved and MODIS emissivity are very similar for most of the pixels. However there are some pixels where the emissivity increases significantly (almost 0.5 ). In order to explain these differences the emissivity image has been compared with other MODIS products: cloud temperature, cloud phase and emissivity. In Fig. 5 the emissivity provided by MODIS is shown, where dark red represents high emissivity ( $\mathrm{e}=1)$ and blue low emissivity. 


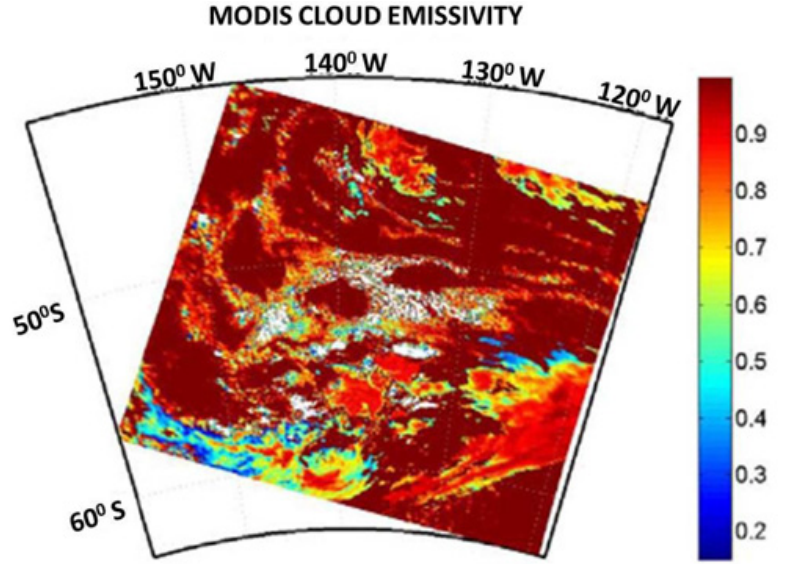

Figure 5. Emissivity provided by MODIS. Dark red represents high emissivity $(\varepsilon=1)$ and dark blue low emissivity $(\varepsilon=0)$.

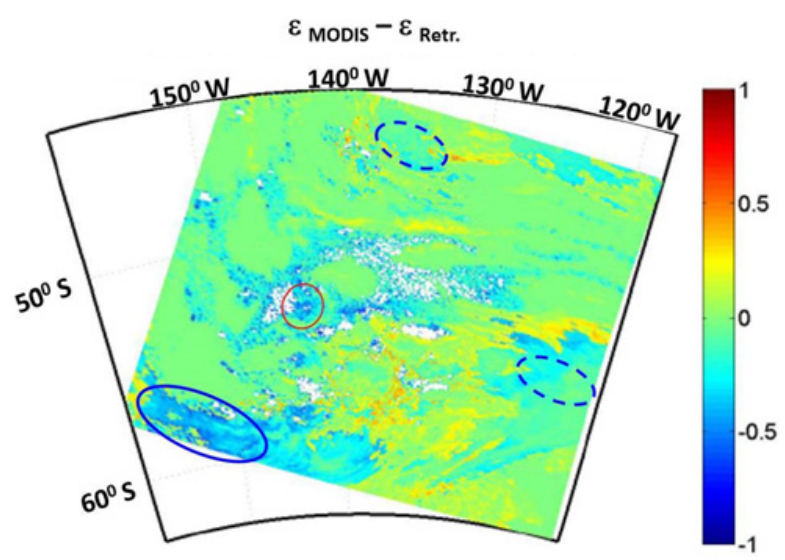

Figure 6. Areas of high emissivity difference. Areas surrounded by solid ovals correspond to high emissivity difference $(-0.5)$. These areas enclose ice pixels (blue) and water pixels (water) but both of them are associated to low emissivity. In the areas enclosed by dashed lines (ice pixels) the emissivity difference is not so high corresponding to higher emissivity.

The comparison of the emissivity difference image with the MODIS products indicates that there is close relationship between the emissivity difference and the emissivity itself, closer than with the rest of the products: cloud temperature and cloud phase. Some areas of the image can be used to illustrate this. These areas are highlighted in Fig. 6.

The solid thick blue oval marks a region where the emissivity difference is high (around -0.5). It corresponds to an ice region so, in principle, the emissivity difference could be attributed to ice phase. However dashed thick blue ovals also enclose ice pixels and the emissivity difference is not so high. Conversely the emissivity difference can be better explained in terms of emissivity. Indeed the area surrounded by the solid thick blue oval shows an emissivity lower than the area enfolded by the dashed thick blue ovals. Moreover, there are also areas of water pixels where the emissivity difference is higher than other water phase areas (pixels encircled by the solid thin red circle). Those areas are also associated to low emissivity pixels (probably broken clouds), which support the statement presented before: the emissivity difference is related to low emissivity areas.

The results of the application of the LUT procedure to real scenarios, i.e., the comparison of the emissivity retrieved by the LUT with the emissivity provided by MODIS, are not completely consistent with the results when the LUT procedure applied to simulated scenarios for low emissivities. It can be due to the nature of the physics problem itself. When the cloud emissivity is low, part of the radiance coming from the elements below the cloud (Earth surface and atmosphere) passes through the cloud and contribute to the radiance received by the sensor. Therefore the BTs calculated from the radiance have a non-negligible contribution of the scenario below the cloud. Since this contribution can be very variable due to the surface characteristics and the atmospherics conditions it could affect significantly the emissivity retrievals. However this and other effects have to be studied in a future work.

\section{Conclusions}

In this work a LUT-based procedure to retrieve the emissivity has been proposed. The emissivity is a key cloud parameter to retrieve the cloud temperature and then the cloud height, which is the aim of the JEM-EUSO IR camera. The LUT has been generated from BTs in $1 \mu \mathrm{m}-$ width bands centred in 10.8 and $12 \mu \mathrm{m}$ since these are the spectral bands of the JEM-EUSO IR camera. These BTs have been simulated in different IR scenarios (different clouds and atmospheric conditions).

The procedure has been validated in simulated scenarios which are different from the scenarios used for the LUT generation. The validation confirms that the LUTbased procedure is able to retrieve the emissivity with good accuracy.

The procedure has also been applied to real scenarios, MODIS images, and the emissivity results have been compared with the emissivity provided by MODIS. The results are very similar except in pixels with low emissivity. However, the percentage of low emissivity pixels is low for both water and ice clouds [6]. It can be attributed to the radiance coming from below the cloud that passes through the cloud and contributes to the BTs. Although this contribution has been considered in the simulations, the high spatial and temporal variability of the temperature and emissivity of the Earth surface makes this contribution very variable. Nevertheless more research is needed to confirm this point.

Concluding: the results obtained with the LUT-based procedure will be very useful for the IR camera images analysis as they will provide the emissivity needed to retrieve the cloud temperature and cloud height of thin clouds. This procedure will be very easy and fast as it does not require ancillary information from other sources, for instance global atmospheric models. Also for this reason the results of the proposed procedure do not depend on possible inaccuracies of ancillary data. Nevertheless considering additional information of other sources (global models, LIDAR, etc.) could improve the final result, which can be a future working line. The results provided by this 
methodology can be combined with the results of other complementary techniques to compare methodologies and also to make the general performance of JEM-EUSO Atmospheric Monitoring System even better.

Authors would like to acknowledge to JEM-EUSO members for supporting encouraging us in this work and letting us to be part of the consortium. JEM-EUSO consortium is being funded by: Basic Science Interdisciplinary Research Projects of RIKEN and JSPS KAKENHI Grant (22340063, 23340081, and 24244042), by the Italian Ministry of Foreign Affairs, General Direction for the Cultural Promotion and Cooperation, by the "Helmholtz Alliance for Astroparticle Physics HAP" funded by the Initiative and Networking Fund of the Helmholtz Association, Germany, and by Slovak Academy of Sciences MVTS JEM-EUSO as well as VEGA grant agency project 2/0081/10. The Spanish Consortium involved in the JEM-EUSO Space Mission is funded by MICINN under projects AYA2009-06037-E/ESP, AYAESP 2010- 19082, AYA2011-29489-C03-01, AYA2011-29489C03-03, AYA2012-39115-C03-01, AYAESP2011- 29489-C03, AYA-ESP2012-39115-C03-02, CSD2009- 00064 (Consolider MULTIDARK) and by Comunidad de Madrid (CAM) under project S2009/ESP-1496.

\section{References}

[1] J.H. Adams et al., Astropart. Phys. 44, 76-90 (2013)

[2] G. Sáez Cano, J.A. Morales de los Ríos, K. Shinozaki, S. Briz, H. Prieto, L. del Peral,
J. H-Carretero, A.J. de Castro, F. Cortés, F. Lopez, A. Neronov, S. Wada, M.D. Rodríguez Frías, for the JEM-EUSO Collaboration, J. Phys.: Conf. Ser. 375 052010 (2012)

[3] A. Neronov, S. Wada, M.D. Rodríguez-Frías, J.A. Morales de los Ríos, G. Sáez Cano,H. Prieto, J. Piñeiro, L. del Peral, J. Hernández, N. Pacheco Gómez, M.D. Sabau, T. Beleguer, C. González, M. Reina, S. Briz, A.J. de Castro, F. Cortés, F. López, G. Herrera, J. Licandro, E. Joven, M. Serra, O.Vaduvescu, A. Anzalone, F.Isgrò, R. Cremonini, C. Cassardo, A. Maurissen, C. Urban, T. Ogawa, K. Shinozaki, "Atmospheric Monitoring System of JEM-EUSO, Proceedings of the 32nd International Cosmic Ray Conference, 6, 338-342 (2011)

[4] S. Toscano, L. Valore, A. Neronov, F. Guarino, F. for the JEM-EUSO Collaboration,Proceedings of the 33rd International Cosmic Ray Conference, 1 143-147 (2013)

[5] M.D. Rodriguez Frias, J. Licandro, M.D. Sabau, M. Reyes,T- Belenguer, M.C. Gonzalez Alvarado, E. Joven, J.A. Morales de los Rios, M SaezPalomino, H. Prieto-Alfonso, G. Saez Cano, J. HCarretero, S. Perez Cano, L. del Peral, for the JEM-EUSO Collaboration. Proceedings of the 33rd International Cosmic Ray Conference, 1, 139-142, (2013)

[6] S. Briz, A.J. de Castro, I. Fernández-Gómez, I. Rodríguez, F. López, J. Appl. Remote Sens., 8, 084990-1 -084990-15 (2014) 